\title{
Use of interferon-gamma release assays in a health care worker screening program: Experience from a tertiary care centre in the United States
}

\author{
Manish Joshi MD FCCP1,2, Thomas P Monson MD², Gail L Woods MD²
}

\begin{abstract}
M Joshi, TP Monson, GL Woods. Use of interferon-gamma release assays in a health care worker screening program: Experience from a tertiary care centre in the United States. Can Respir J 2012;19(2):84-88.
\end{abstract}

BACKGROUND: Interferon-gamma release assays including the QuantiFERON-TB Gold In-Tube test (QFT-GIT [Cellestis Ltd, Australia]) may be used in place of the tuberculin skin test (TST) in surveillance programs for Mycobacterium tuberculosis infection control. However, data on performance and practicality of the QFT-GIT in such programs for health care workers (HCWs) are limited.

OBJECTIVES: To assess the performance, practicality and reversion rate of the QFT-GIT among HCWs at a tertiary health care institution in the United States.

METHODS: Retrospective chart review of HCWs at Central Arkansas Veterans Healthcare System (Arkansas, USA) who underwent QFT-GIT testing as a part of their employee screening between November 1, 2008 and October 31, 2009.

RESULTS: QFT-GIT was used to screen $3290 \mathrm{HCW}$. The initial QFT-GIT was interpreted as positive for $129(3.9 \%) \mathrm{HCW}$, negative for $3155(95.9 \%)$ and indeterminate for six $(0.2 \%)$. Testing with QFTGIT was repeated in $45 \mathrm{HCW}$ sho had positive results on the initial test. The QFT-GIT reverted to negative in $18(40.0 \%) \mathrm{HCW}$, all of whom had negative TST status and initial interferon-gamma values of $0.35 \mathrm{IU} / \mathrm{mL}$ to $2.0 \mathrm{IU} / \mathrm{mL}$.

CONCLUSIONS: The QFT-GIT test is feasible in large health care setting as an alternative to TST for $M$ tuberculosis infection screening in HCWs but is not free from challenges. The major concerns are the high number of positive test results and high reversion rates on repeat testing, illustrating poor short-term reproducibility of positive QFT-GIT test results. These results suggest adopting a borderline zone between interferon-gamma values of $0.35 \mathrm{IU} / \mathrm{mL}$ to $2.0 \mathrm{IU} / \mathrm{mL}$, and cautious clinical interpretation of values in this range.

Key Words: Health care workers; Interferon-gamma release assays; Tuberculosis; QuantiFERON Gold In-Tube assay

$\mathrm{H}$ ealth care workers $\left(\mathrm{HCW}_{\mathrm{s}}\right)$ are at increased risk of becoming infected with Mycobacterium tuberculosis through occupational exposure. Periodic screening of HCWs for $\mathrm{M}$ tuberculosis infection (MTBI) is a common practice and an essential component of many hospital infection control programs in the low tuberculosis (TB) prevalence countries including Canada and the United States (1). HCWs are typically screened with a tuberculin skin test (TST) and for symptoms suggestive of TB. Those with symptoms or a positive TST undergo additional testing that typically includes a chest $\mathrm{x}$-ray. Latent $\mathrm{M}$ tuberculosis infection (LTBI) is diagnosed based on a positive TST result after TB disease has been excluded. The TST has many disadvantages including the assessment of response to a complex mix of antigens included in tuberculin-purified protein derivative that may cross-react following Bacille Calmette-Guérin (BCG) vaccination or exposure to non-TB mycobacteria, technical variations with administration, required return visits for reading and variability in interpretation of test results (2).

\author{
L'utilisation des tests de libération d'interféron \\ gamma dans un programme de dépistage chez des \\ travailleurs de la santé : l'expérience d'un centre de \\ soins tertiaires des États-Unis
}

HISTORIQUE : Les tests de libération d'interféron gamma, y compris le
test QuantiFERON-TB Gold In-Tube (QFT-GIT [Cellestis Ltd, Australie]),
pourraitent peut-être remplacer le test cutané à la tuberculine (TCT) dans
les programmes de surveillance pour contrôler l'infection au Mycobacterium
tuberculosis. Cependant, les données sur le rendement et le caractère pra-
tique du test QFT-GIT dans de tels programmes destinés aux travailleurs de
la santé (TdS) sont limités. OBJECTIFS : Évaluer le rendement, le caractère pratique et le taux de réversion du test de QFT-GIT chez les TdS d'un établissement de soins tertiaires des États-Unis.

MÉTHODOLOGIE : Analyse rétrospective des dossiers des TdS du Central Arkansas Veterans Healthcare System de l'Arkansas, aux États-Unis, qui ont subi le test QFT-GIT dans le cadre du dépistage des employés entre le $1^{\text {er }}$ novembre 2008 et le 31 octobre 2009.

RÉSULTATS : Le test QFT-GIT a servi au dépistage de 3290 TdS. Le test QFT-GIT initial a été interprété comme positif auprès de 129 TdS (3,9\%), comme négatif chez 3155 TdS (95,9\%) et comme indéterminé chez six TdS (0,2\%). Le test QFT-GIT a été repris chez $45 \mathrm{TdS}$ qui avaient obtenu des résultats positifs au test initial. Il a donné des résultats négatifs chez 18 TdS (40,0\%), qui avaient tous obtenu un TCT négatif et des valeurs initiales d'interféron gamma de $0,35 \mathrm{UI} / \mathrm{mL}$ à $2,0 \mathrm{UI} / \mathrm{mL}$.

CONCLUSIONS : Le test QFT-GIT est faisable dans de grands établissements de santé pour remplacer le TCT dans le dépistage du M tuberculosis chez les TdS, mais il n'est pas sans défis. Les principales préoccupations sont le nombre élevé de résultats faux-positifs et le taux élevé de réversion à la reprise du test, qui démontre une mauvaise reproductibilité à court terme des résultats positifs du test QFT-GIT. Ces résultats incitent à préconiser l'adoption d'une zone seuil entre les valeurs d'interféron gamma de $0,35 \mathrm{UI} / \mathrm{mL}$ à $2,0 \mathrm{UI} / \mathrm{mL}$ et une interprétation clinique prudente des valeurs faisant partie de cette plage.

Interferon-gamma (IFN- $\gamma$ ) release assays (IGRAs) are in vitro tests for MTBI that can be used in place of TST to screen HCWs (3). Two IGRAs are commercially available for the detection of MTBI in the United States including the QuantiFERON-TB Gold In-Tube test (QFT-GIT [Cellestis Ltd, Australia]) and the T-SPOT.TB assay (Oxford Immunotec, United Kingdom) (2,3). QFT-GIT assess immunological responsiveness to specific $M$ tuberculosis proteins including early secretory antigenic target-6 (ESAT-6), culture filtrate protein 10 (CFP10) and TB 7.7, which offers several advantages over the TST (2). These antigens do not cross-react with BCG or with most non-TB mycobacteria. The QFT-GIT test requires a single patient visit $(2,3)$.

The Centers for Disease Control and Prevention (CDC, Georgia, USA) published guidelines for using IGRAs and indicated that certain IGRAs, including QFT-GIT may be used in surveillance programs for MTBI including those for HCWs (3). On the contrary, the most

${ }^{1}$ Pulmonary and Critical Care Division, University of Arkansas for Medical Sciences; ${ }^{2}$ Central Arkansas Veterans Healthcare System, Little Rock, Arkansas, USA

Correspondence: Dr Manish Joshi, University of Arkansas for Medical Sciences, Central Arkansas Veterans Healthcare System, 4300 West 7th Street, 5C 144, Little Rock, Arkansas 72205, USA. Telephone 501-257-5786, fax 501-686-7893, e-mail manish.joshi@va.gov 
recent 2010 Canadian guidelines (4) do not recommend the use of IGRAs as a baseline test in serial testing of HCWs. However, data regarding the performance of the QFT-GIT in such screening programs for HCWs in the United States are limited $(2,3)$.

The Central Arkansas Veterans Healthcare System (CAVHS) is a tertiary care academic Veterans Administration hospital affiliated with the University of Arkansas for Medical Sciences (Arkansas, USA) employing more than 3000 individuals. Screening of HCWs for MTBI with the TST, initially at the time of recruitment and annually thereafter, was the standard practice at CAVHS for years following the published guidelines (1); the annual TST conversion rate in the five years before 2008 was less than $0.1 \%$. In November 2008, CAVHS began to use the QFT-GIT test in place of TST for screening both employees and patients for MTBI. After this change, CAVHS were confronted with an unexpectedly high number of positive QFT-GIT test results ( $>20$-fold higher than baseline positive TST results). In the first four weeks, five of $210 \mathrm{HCWs}(2.5 \%)$ tested positive with the QFT-GIT test. Active TB disease was ruled out by symptom assessment and chest radiographs. This resulted in a clinical decision-making dilemma of whether to offer LTBI treatment because all of the HCWs who tested positive on QFT-GIT testing had a negative TST history. All five of these HCWs underwent repeat testing with QFT-GIT and TST. Not only did all five revert to negative QFT-GIT, but their TST status also remained negative. There was no available literature or data to guide us in this unique situation. Because of positive QFT-GIT results, the infection control team at CAVHS decided to offer repeat QFT-GIT tests to all HCWs whose initial QFT-GIT was positive. No repeat testing was performed on $\mathrm{HCW}$ s with negative QFT-GIT results. The aim of the present study was to assess the performance and practicality of use, and the reversion rates for QFT-GIT testing among $\mathrm{HCW}$ sho tested positive in an employee screening program in the United States.

\section{METHODS}

The present study was a retrospective chart review conducted at the CAVHS in Little Rock, Arkansas. The study was initiated after approval by the CAVHS Institutional Review Board and Research and Development Committee. Electronic medical records of all HCWs were queried to identify those who had a positive QFT-GIT as a part of their employee screening between November 1, 2008 and October 31, 2009. Data regarding age, sex, previous TST results and all QFT-GIT results for those with positive QFT-GIT results were collected. All HCWs underwent screening for MTBI with QFT-GIT, irrespective of previous TST status, to establish baseline test results for comparison with future test results.

QFT-GIT was performed according to the manufacturer's instructions (5). Briefly, approximately $1 \mathrm{~mL}$ of blood is collected directly into each of three tubes (nil, TB antigen and mitogen control). The tubes are then shaken vigorously and transported to the clinical laboratory. Once the tubes reach the microbiology section, they are again mixed by shaking and then immediately incubated at a mean $( \pm$ SD) temperature of $37 \pm 1^{\circ} \mathrm{C}$. The time from collection to incubation is approximately $1 \mathrm{~h}$. After incubation in ambient air for $16 \mathrm{~h}$ to $24 \mathrm{~h}$, the tubes are centrifuged for $15 \mathrm{~min}$ at $2500 \mathrm{rpm}$ to separate cells from plasma. Freshly prepared conjugate, then plasma is added to an ELISA plate. The plate is placed on a microplate shaker to ensure mixing of the conjugate and plasma, covered, incubated at room temperature for $120 \pm 5 \mathrm{~min}$, then washed using a Columbus semi-automated ELISA plate washer (Tecan Group Ltd, Switzerland). Enzyme substrate is added to each well and the plates are mixed again, covered and incubated at room temperature for $30 \mathrm{~min}$. Enzyme stop solution is added, and the optical density of each well is determined within $5 \mathrm{~min}$ of stopping the reaction using a Sunrise ELISA plate reader (Tecan Group Ltd, Switzerland).

The test results were reported positive if the IFN- $\gamma$ value of TB antigen minus nil was $\geq 0.35 \mathrm{IU} / \mathrm{mL}$ and negative if $<0.35 \mathrm{IU} / \mathrm{mL}$. Test results were considered to be indeterminate if nil concentrations were $>8.0 \mathrm{IU} / \mathrm{mL}$ and mitogen differences were $<0.5 \mathrm{IU} / \mathrm{mL}$, as per manufacturer's guidelines (5). In the present study, QFT-GIT reversion was defined as initial positive test results becoming negative on subsequent testing.

\section{Statistical analysis}

Statistical analysis was conducted using SAS version 9.2 (SAS Institute, USA). All reported $\mathrm{P}$ values were two-tailed and calculated with statistical significance set at $\mathrm{P} \leq 0.05$. The $\chi^{2}$ test was used to compare categorical variables and independent group $t$ tests were used to compare continuous variables. For comparison of TB responses, values $>10 \mathrm{IU} / \mathrm{mL}$ were replaced with a value of $10 \mathrm{IU} / \mathrm{mL}$.

\section{RESULTS}

A total of $3290 \mathrm{HCW}$ s underwent a QFT-GIT test between November 1, 2008 and October 31, 2009. The initial QFT-GIT test was interpreted as positive for $129(3.9 \%) \mathrm{HCW}$, negative for 3155 (95.9\%) HCWs, and indeterminate for six (0.2\%) HCWs (Table 1). The characteristics of the $129 \mathrm{HCW}$ s who tested positive on QFT-GIT testing are summarized in Table 2 . The mean $( \pm$ SD) age of the 129 HCWs who tested positive with QFT-GIT was $49.9 \pm 12.2$ years. In this population, $70(54.2 \%)$ were males. Among the $129 \mathrm{HCW}$ s with positive QFT-GIT test results, $53(41 \%)$ had a history of positive TST, 62 (48\%) had a history of negative TST(s) and 14 (11\%) had unknown TST status.

Testing with QFT-GIT was repeated (within two to 30 days) in $45(34.9 \%) \mathrm{HCW}$ s who were positive on initial testing. The repeat QFT-GIT results and TST status of these HCWs are shown in Figure 1. The test reverted to negative in $18(40.0 \%)$ of the $45 \mathrm{HCW}$ who underwent repeat QFT-GIT. Among HCWs whose QFT-GIT reverted to negative, the mean and median TB response from the initial QFTGIT were $1.44 \mathrm{IU} / \mathrm{mL}$ and $1.05 \mathrm{IU} / \mathrm{mL}$, respectively. Changes in $\mathrm{TB}$ responses for these $18 \mathrm{HCW}$ s are shown in Figure 2. The initial values were distributed over a range of $0.36 \mathrm{IU} / \mathrm{mL}$ to $2.1 \mathrm{IU} / \mathrm{mL}$ (with one outlier not shown in this figure with a value $\geq 10 \mathrm{IU} / \mathrm{mL}$ ) whereas the $\mathrm{TB}$ responses obtained on repeat testing were clustered in a much smaller range $(-0.1$ to 0.1$)$. While none of the subjects whose QFTGIT reverted to negative had a previous positive TST, 13 (48\%) of 27 subjects whose QFT-GIT remained positive had a previous positive TST. Of the HCWs with consistently positive QFT-GITs, 13 (48\%) had a negative TST history, and the TST status was unknown for one individual (Figure 1). The IFN- $\gamma$ values on repeat QFT-GIT testing increased in 10 of $13 \mathrm{HCW}$ s who had previous positive TST, and decreased in 10 of $13 \mathrm{HCW}$ s who had negative TST history.

$\mathrm{TB}$ response values were compared for the $18 \mathrm{HCW}$ who reverted on repeat testing with the values for the 27 who remained positive on repeat testing (Figure 3 ). The mean initial TB response in the group who reverted was $1.44 \mathrm{IU} / \mathrm{mL}$ ( $95 \%$ CI $0.36 \mathrm{IU} / \mathrm{mL}$ to $2.52 \mathrm{IU} / \mathrm{mL}$ ), whereas the mean initial TB response in the group who remained positive was $3.47 \mathrm{IU} / \mathrm{mL}$ (95\% CI $1.99 \mathrm{IU} / \mathrm{mL}$ to $4.95 \mathrm{IU} / \mathrm{mL}[\mathrm{P}=0.02]$ ). When limited to HCWs whose previous TST was negative (ie, those typically retest in employee screening programs), the difference in TB response values among those who reverted versus those who remained positive was not statistically significant $(\mathrm{P}=0.84)$ (Figure 4).

\section{DISCUSSION}

To our knowledge, the present study was the first to be presented and published as a scientific abstract (6) to assess the performance and practicality of the QFT-GIT test in a substantial number of United States HCWs undergoing screening for MTBI in a large tertiary centre. Although we found QFT-GIT testing feasible in a large tertiary health care setting, and logistically more convenient to perform than TST, we were faced with many unique challenges and clinical situations after replacing the TST with the QFT-GIT test. We had an unexpectedly high number of positive QFT-GIT test results (>20-fold higher than baseline positive TST results). This not only led to additional testing, including chest $\mathrm{x}$-rays, TST and repeat QFT-GIT, but also lack of confidence in test results among our employees. Moreover, 
TABLE 1

QFT-GIT* test results and tuberculin skin test (TST) status of 3290 health care workers who underwent QFT-GIT testing in a one-year study period

\begin{tabular}{lcccc}
\hline & \multicolumn{3}{c}{ TST history } & \\
\cline { 2 - 4 } QFT-GIT & Positive & Negative & Unknown & Total \\
\hline Positive & 53 & 62 & 14 & 129 \\
Negative & 78 & 2474 & 603 & 3155 \\
Indeterminate & 0 & 6 & 0 & 6 \\
Total & 131 & 2542 & 617 & 3290 \\
\hline
\end{tabular}

Data presented as n. *QuantiFERON-TB Gold In-Tube test (Cellestis Ltd, Australia)

TABLE 2

Characteristics of 129 health care workers (HCWs) who tested positive on initial QFT-GIT* test

\begin{tabular}{lc}
\hline Characteristic & HCWs \\
\hline Sex & $59(46)$ \\
$\quad$ Female & $70(54)$ \\
$\quad$ Male & \\
Tuberculin skin test history & $62(48)$ \\
Negative & $53(41)$ \\
Positive & $14(11)$ \\
Unknown & $49.9 \pm 12.2$ \\
Age, years, mean \pm SD &
\end{tabular}

Data presented as $n$ (\%) unless otherwise indicated. *QuantiFERON-TB Gold In-Tube test (Cellestis Ltd, Australia)

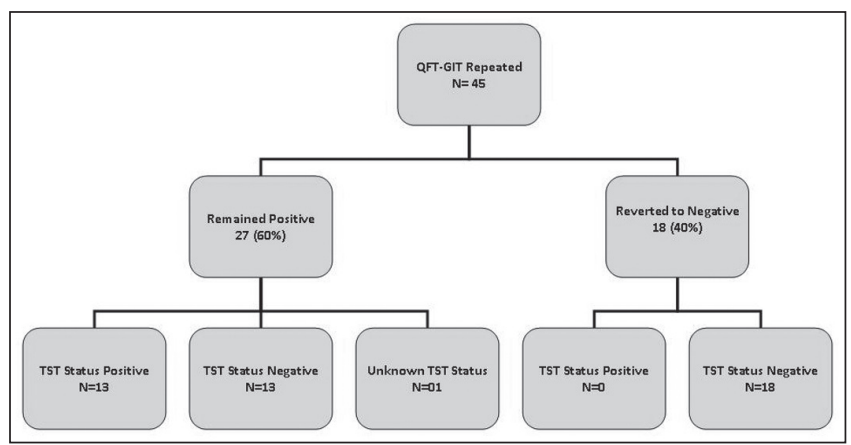

Figure 1) The QuantiFERON-TB Gold In-Tube (QFT-GIT [Cellestis Ltd, Australia]) test results and history of tuberculin skin test (TST) status in 45 health care workers who underwent repeat testing

this resulted in a major dilemma when it came to clinical decision making in terms of offering LTBI treatment because many of these HCWs who tested positive on QFT-GIT testing had negative TST(s) in the preceding years. Interestingly, our results were very similar to another study recently published by Gandra et al (7), in which the authors raise a similar concern with serial QFT-GIT testing of HCWs.

Another concerning issue with serial QFT-GIT testing is shortterm reproducibility, as demonstrated by the high reversion rate (40\%) in our study population. The mean IFN- $\gamma$ value for these HCWs who underwent repeat QFT-GIT testing was $1.44 \mathrm{IU} / \mathrm{mL}$. Our results agree with previous studies questioning the short-term reproducibility of QFT-GIT (2,8-12), but our mean IFN- $\gamma$ values were higher than those previously reported in literature. It has been proposed in these studies to increase the cut-off value for a positive test result to $0.70 \mathrm{IU} / \mathrm{mL}$, but our results do not support this value for many reasons. First, our mean initial IFN $-\gamma$ values for $\mathrm{HCW}$ s who reverted to negative was $1.44 \mathrm{IU} / \mathrm{mL}$ and is two times higher than the proposed $0.70 \mathrm{IU} / \mathrm{mL}$. Hence, raising the cut-off value to $0.70 \mathrm{IU} / \mathrm{mL}$ will not solve the problem of these likely false-positive results. Second, a fixed cut-off in this population will continue to pose challenges because of test (QFT-GIT) variability

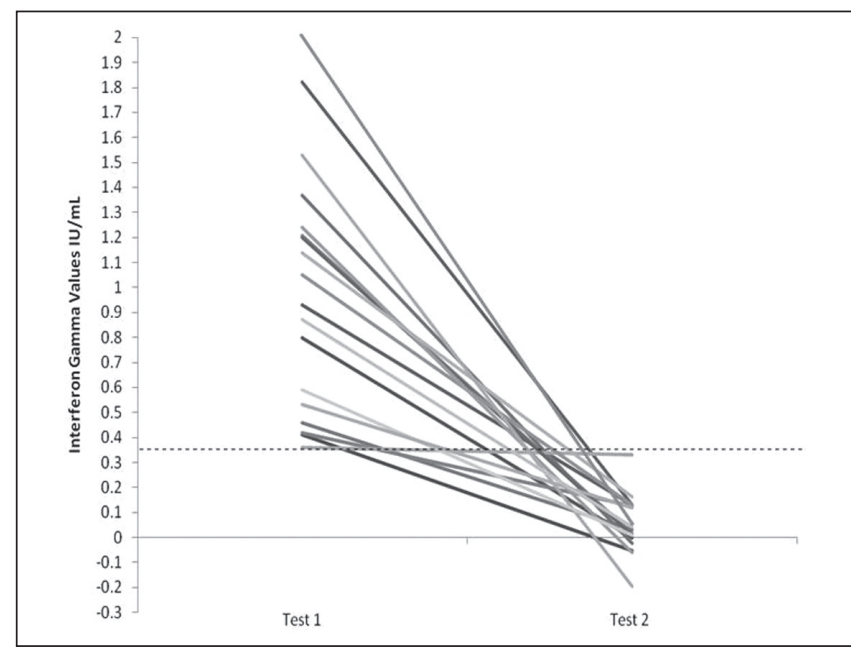

Figure 2) The initial and repeat interferon (IFN)-gamma values in 18 health care workers who reverted to negative on repeat QuantiFERON-TB Gold In-Tube (QFT-GIT [Cellestis Ltd, Australia]) testing. The dotted line represents the cut-off IFN-gamma value for positive tests $(0.35 \mathrm{IU} / \mathrm{mL})$. An outlier with IFN-gamma value $\geq 10$ is not represented in this figure

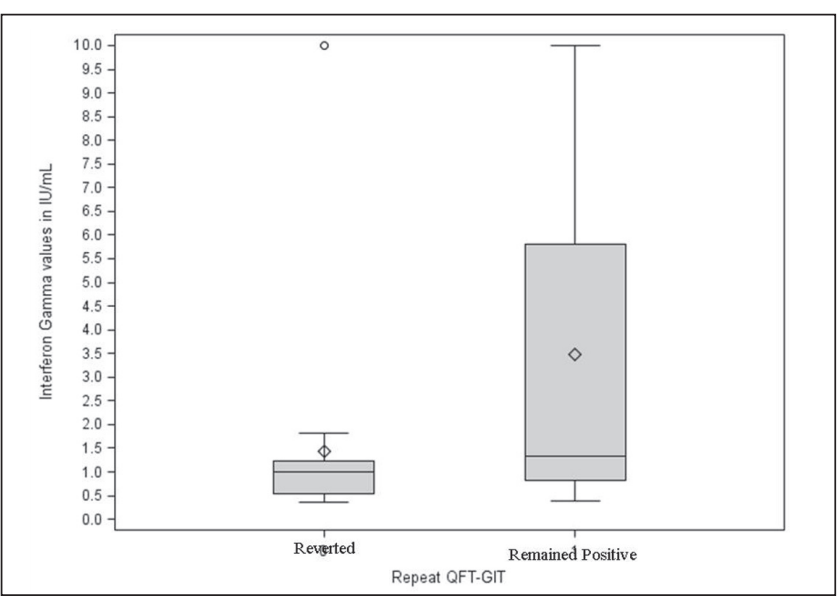

Figure 3) Box-and-whisker plots showing distribution of initial interferongamma values in health care workers whose repeat QuantiFERON-TB Gold In-Tube test (QFT-GIT [Cellestis Ltd, Australia]) result reverted to negative $(n=18)$ versus those who remained positive $(n=27)$. On comparing interferon-gamma values, there was a significant difference $(P=0.02)$ in the mean levels between the two groups

in serial testing, and it is not possible to distinguish false positives from true positives without having a gold standard for LTBI diagnosis. To address this problem, we suggest adding a borderline zone for interpretation of test results in serial testing of HCWs when IFN- $\gamma$ values are between $0.35 \mathrm{IU} / \mathrm{mL}$ to $2.0 \mathrm{IU} / \mathrm{mL}$. We also recommend cautious clinical interpretation of QFT-GIT test results in this borderline zone. Repeat testing with QFT-GIT and/or TST should be considered for HCWs whose IFN- $\gamma$ values are in the above borderline range and TST status is negative. More longitudinal studies investigating positive predictive value and likelihood of developing active TB after a positive QFT-GIT test are needed to address this issue of optimal borderline zone in this subset of the population.

The reason(s) for the high reversion rates and, thus, poor shortterm reproducibility is unclear. It may be due to variation in host immune responses, although this is perplexing when the QFT-GIT result changes from positive to negative within a few days. Another possibility is pretest variability as a result of the recommended manufacturer's test protocol. To investigate this, personnel collecting the specimens and those performing the test were closely observed during 
testing of a few samples. We found that in several cases of positive test results (not reported as final results in the present study), pretest variability was a contributing factor for these likely false-positive results. According to the manufacturer's instuctions, tubes should be shaken vigorously once blood is collected (5). We noted the force with which tubes were shaken varied among phlebotomists. Vigorous shaking was often associated with a false-positive result. Once this was recognized, phlebotomists were retrained, emphasizing proper (ie, not so vigorous) shaking. The number of false-positive results then declined but still occurred despite following the manufacturer's exact directions to perform the QFT-GIT test, suggesting that other factor(s) are involved. Further studies are needed, both in a controlled research setting and in the real world, to fully understand QFT-GIT variability and reproducibility. We would certainly recommend not shaking the test tubes too vigorously and would also suggest to the manufacturer to add a standardized shaking device to the test kit.

The comparison of mean IFN- $\gamma$ values in HCWs who reverted to negative and those who remained positive on repeat QFT-GIT testing showed statistical significance. However, when these two groups were compared, including only the HCWs who had negative TST status (ie, those typically retested in employee screening programs), the comparison of mean IFN $-\gamma$ values showed no statistical significance. This implies that negative TST status is a comparable (with QFT-GIT) predictor of absence of LTBI in serial testing. The diagnosis of LTBI among HCWs should be made using a combination of epidemiological and other diagnostic methods, and not based on TST results and/or QFT-GIT results alone.

We had another interesting observation in our results. The mean IFN- $\gamma$ values on repeat QFT-GIT testing increased in $\mathrm{HCW}$ s with a history of positive TST, whereas they decreased in HCWs with negative TST history. It is yet to be determined as to what absolute difference in these IFN- $\gamma$ values is actually clinically relevant in serial testing (13). It is, therefore, difficult to extrapolate anything conclusive; however, these trends are interesting to note and should be explored in future research. Nevertheless, we agree with Pai et al (13), who recommend that health professionals who interpret serial QFT-GIT should not merely rely on dichotomous (positive/negative) results, but learn to interpret trends in IFN- $\gamma$ values over time.

The first and most recent systemic review of IGRAs in HCW by Zwerling et al (14) addressed many challenges involved in serial testing. The review concluded that the current guidelines and evidence available on the use of IGRAs needs an update to adequately address the challenges raised by serial testing.

Our study had several limitations. First, it was retrospective in nature. Repeat QFT-GIT testing was performed only in HCWs whose initial test results were positive. The negative tests were assumed to be true negatives despite 60\% discordance among TST and QFT-GIT. Second, not all of the HCWs who had positive QFT-GIT test results underwent repeat QFT-GIT testing, which could have influenced reversion rates. Third, concurrent TSTs were not performed in all HCWs who underwent repeat QFT-GIT testing. Another limitation is that we did not have BCG data on all employees. However, being a federal facility, most of our employees are US-born citizens with a negative BCG history.

\section{CONCLUSION}

The QFT-GIT is not yet ready for 'prime time' to screen HCWs given the high number of positive test results and high reversion rates on repeat testing. The current CDC guidelines (15) supporting QFT-GIT test to screen HCWs for MTBI are based on evidence from controlled research studies and need an update in view of our current translational research. We hope that our large study and practical experience with QFT-GIT in the real world will help many health care organizations that are facing the same challenges that we encountered when TST was replaced by QFT-GIT to screen HCWs for LTBI. It will also guide the institutions who are in the process of implementing QFTGIT. Finally, additional research studies are needed to assess biological

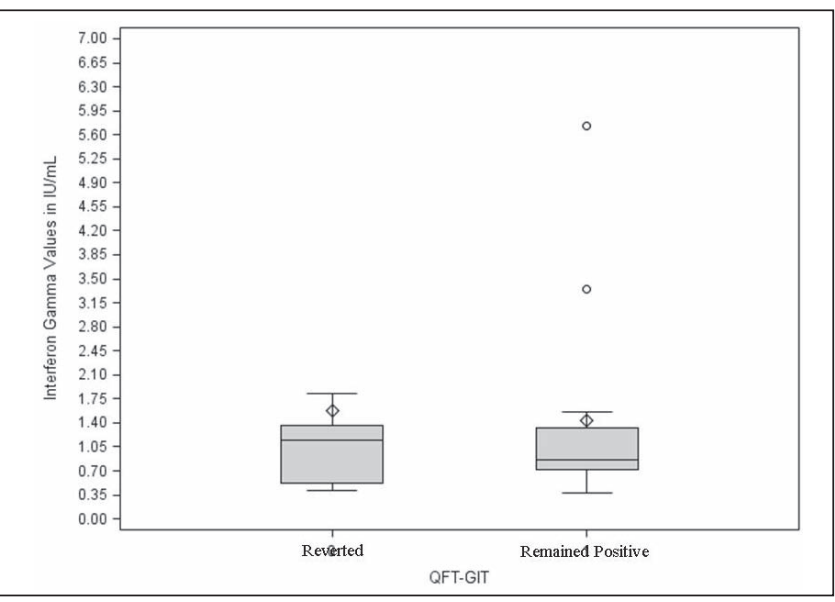

Figure 4) Box-and-whisker plots showing distribution of initial interferongamma values in health care workers who underwent repeat QuantiFERON-TB Gold In-Tube testing (QFT-GIT [Cellestis Ltd, Australia]) testing and had 'negative' tuberculin skin test status. On comparing interferon-gamma values, there was no significant difference $(P=0.84)$ in the mean levels among the health care workers whose repeat QFT-GIT test results reverted from positive to negative $(n=18)$ versus those that remained positive $(n=13)$

variability, reproducibility and the likelihood of developing active TB after a positive QFT-GIT test in low-TB incidence countries.

ACKNOWLEDGEMENTS: The authors thank Dr Gerald H Mazurek MD, Division of Tuberculosis Elimination, CDC for critical review of our manuscript. They also thank Dr Anita Joshi BDS MPH, for critical review of our results and statistical section of the manuscript.

DISCLOSURES: The authors have no financial disclosures or conflicts of interest to declare.

\section{REFERENCES}

1. Jensen PA, Lambert LA, Iademarco MF, Ridzon R. Guidelines for preventing the transmission of Mycobacterium tuberculosis in health-care settings, 2005. MMWR Recomm Rep 2005;54:1-141.

2. Menzies D, Pai M, Comstock G. Meta-analysis: New tests for the diagnosis of latent tuberculosis infection: Areas of uncertainty and recommendations for research. Ann Intern Med 2007;146:340-54.

3. Mazurek GH, Jereb J, LoBue P, Iademarco MF, Metchock B, Vernon A; Division of Tuberculosis Elimination, National Center for HIV, STD, and TB Prevention, Centers for Disease Control and Prevention (CDC). Guidelines for using the QuantiFERON-TB Gold test for detecting Mycobacterium tuberculosis infection, United States. MMWR Recomm Rep 2005;54(RR-15):49-55.

4. Canadian Tuberculosis Committee (CTC). Recommendations on interferon gamma release assays for the diagnosis of latent tuberculosis infection - 2010 update. Can Commun Dis Rep 2010;36:1-22.

5. Cellestis: QuantiFERON ${ }^{\circledR}$-TB Gold In-Tube package insert. $<$ www.cellestis.com/IRM/content/pdf/QuantiFeron\%20US\%20 VerH_Jul2010.pdf $>$ (February 21, 2011).

6. Joshi M, Monson T, Woods G. Practical Experience With the QFT-GIT assay for LTBI annual testing among US health-care workers in a large tertiary setting. Chest 2010;138:746A (Abst).

7. Gandra S, Scott W, Somaraju V, Wang H, Wilton S, Feigenbaum M. Questionable effectiveness of the QuantiFERON-TB Gold Test (Cellestis) as a screening tool in healthcare workers. Infect Control Hosp Epidemiol 2010;31:1279-85.

8. Perry S, Sanchez L, Yang S, Agarwal Z, Hurst P, Parsonnet P. Reproducibility of QuantiFERON-TB Gold In-Tube assay. Clin Vaccine Immunol 2008;15:425-32.

9. Pai M, Joshi R, Dogra S, et al. Serial testing of health care workers for tuberculosis using interferon-gamma assay. Am J Respir Crit Care Med 2006;174:349-55. 
10. van Zyl-Smit RN, Zwerling A, Dheda K, Pai M. Within-subject variability of interferon-g assay results for tuberculosis and boosting effect of tuberculin skin testing: A systematic review.

PLoS OneE 2009;4:e8517.

11. Ringshausen F, Nienhaus A, Costa JT, et al. Within-subject variability of Mycobacterium tuberculosis-specific interferon-gamma responses in German health care workers. Clin Vaccine Immunol 2011;18:1176-82

12. Veerapathran A, Joshi R, Goswami K, et al. T-cell assays for tuberculosis infection: Deriving cut-offs for conversions using reproducibility data. PLoS ONE 2008;3:e1850.
13. Pai M, O'Brien R. Serial testing for tuberculosis: Can we make sense of T cell assay conversions and reversions? PLoS Med 2007;4:e208.

14. Zwerling A, van den hof S, Scholten J, Cobelens F, Menzies d, Pai M. Interferon-gamma release assays for tuberculosis screening of healthcare workers: A systematic review. Thorax 2012;67:62-70.

15. Mazurek M, Jereb J, Vernon A, LoBue P, Goldberg S, Castro K. Updated guidelines for using interferon Gamma release assays to detect Mycobacterium tuberculosis infection in the United States. MMWR Recomm Rep 2010;59:1e25. 


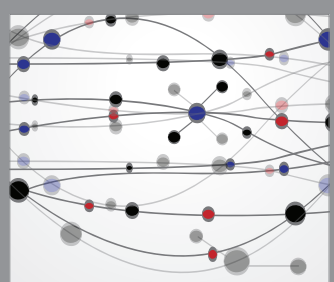

The Scientific World Journal
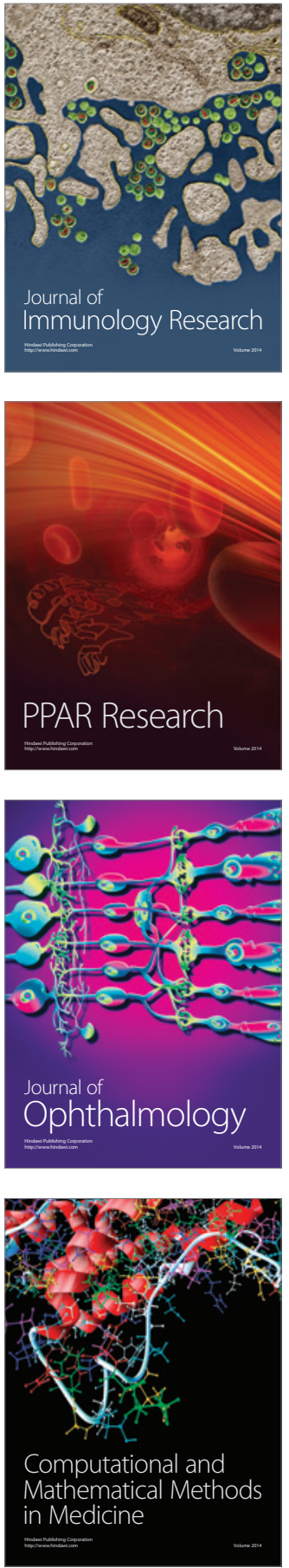

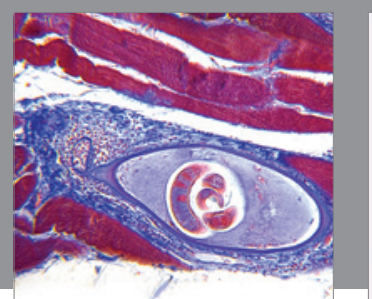

Gastroenterology Research and Practice

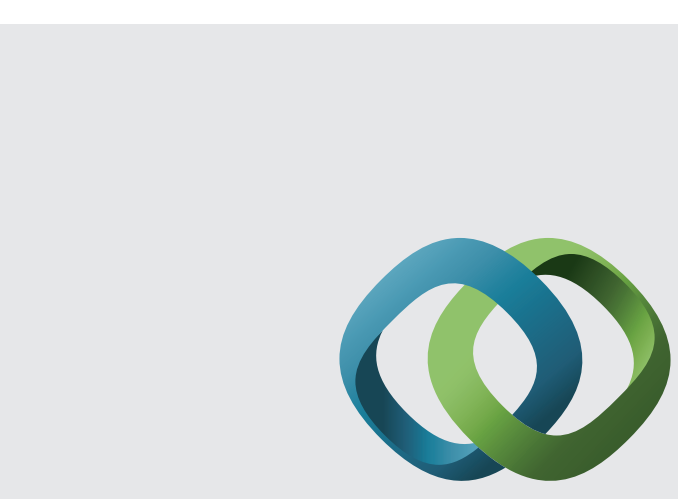

\section{Hindawi}

Submit your manuscripts at

http://www.hindawi.com
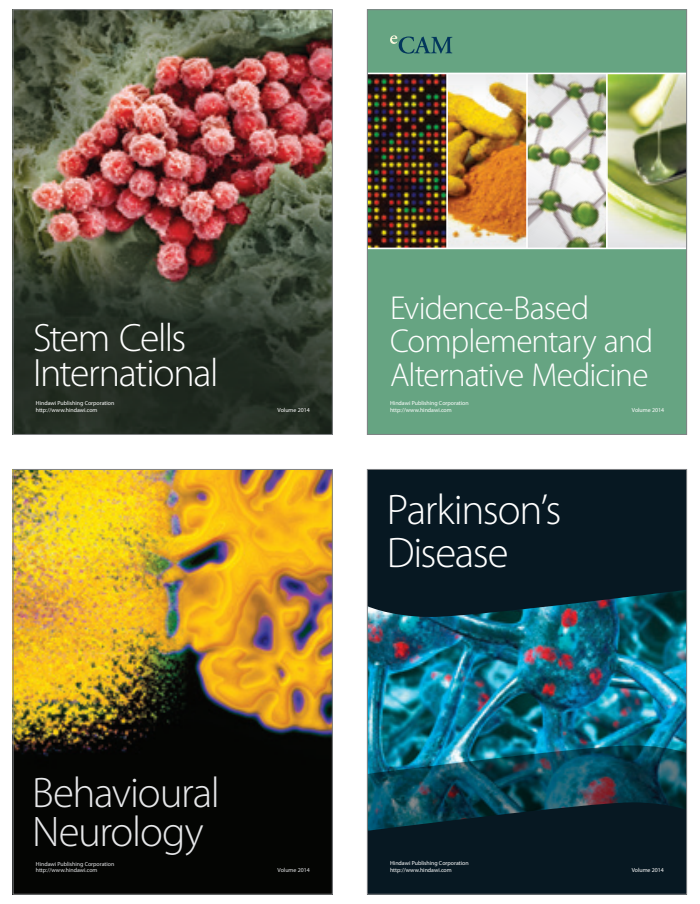
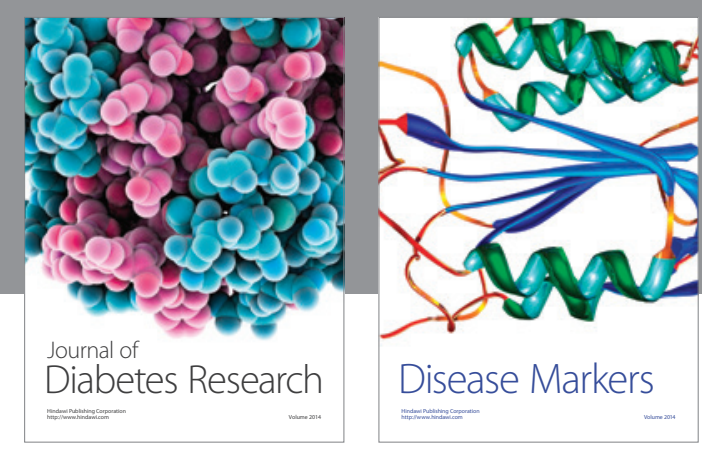

Disease Markers
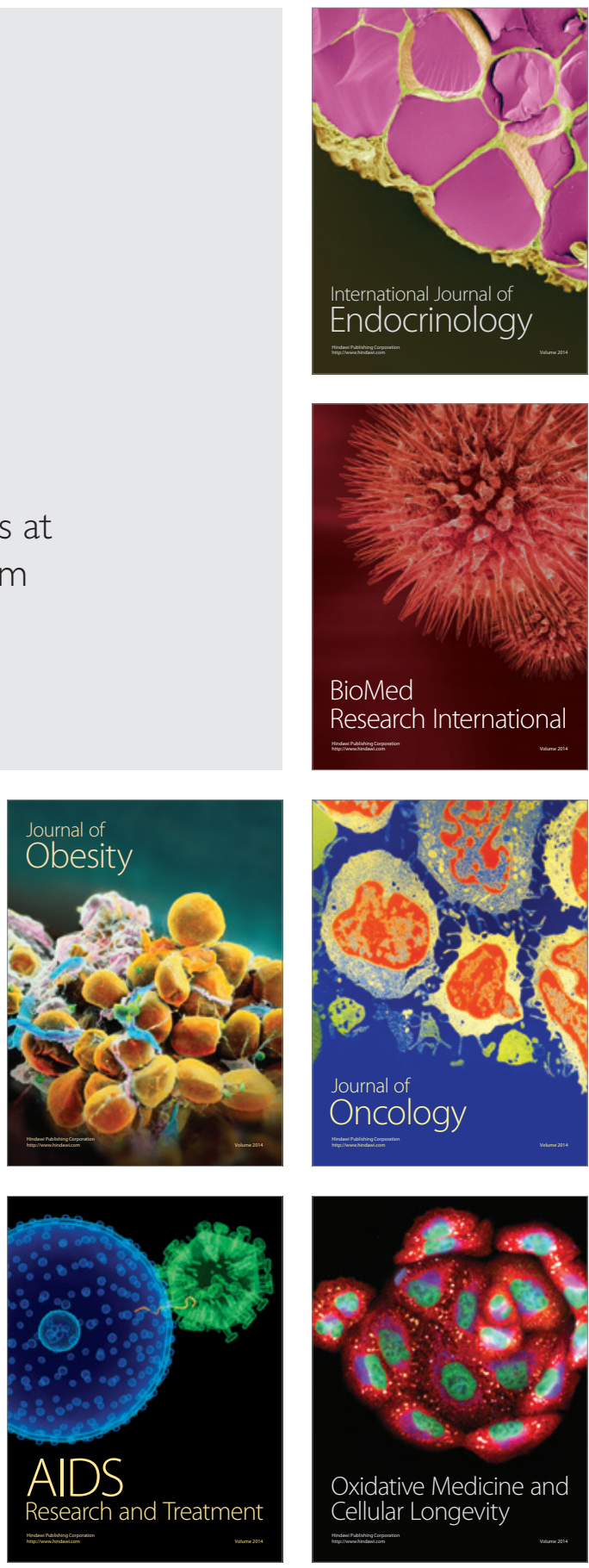